\title{
N-3 Polyunsaturated Fatty Acids, Body Fat and Inflammation
}

\author{
Anne-Sofie Q. Lund ${ }^{\text {a* }}$ Ann Louise Hasselbalch ${ }^{\text {a* }}$ Michael Gamborg ${ }^{\text {a }}$ \\ Kristin Skogstrand $^{b}$ David M. Hougaard ${ }^{b}$ Berit L. Heitmann ${ }^{c}$ \\ Kirsten O. Kyvik $^{d} \quad$ Thorkild I.A. Sørensen $^{a} \quad$ Tine Jess $^{a}$ \\ ${ }^{a}$ Institute of Preventive Medicine, Copenhagen University Hospital, ${ }^{b}$ Department of \\ Clinical Biochemistry and Immunology, Statens Serum Institute, 'Research Unit for Dietary \\ Studies, Institute of Preventive Medicine, dinstitute of Regional Health Services Research \\ and the Danish Twin Registry, Institute of Public Health, University of Southern Denmark, \\ Copenhagen, Denmark
}

\section{Key Words}

Anthropometry $\cdot$ Fat mass $\cdot$ Inflammation $\cdot$ Polyunsaturated fatty acids

\begin{abstract}
Background: Based on animal studies, n-3 polyunsaturated fatty acids (PUFAs) have been suggested to lower the risk of obesity and inflammation. We aimed to investigate if, among humans, intake of n-3 PUFAs was associated with i) total body fat, ii) body fat distribution and iii) obesity-related inflammatory markers. Methods: The study population consisted of 1,212 healthy individuals with information on habitual food intake from food frequency questionnaires, six different measures of body fat, and levels of six circulating inflammatory markers. Multiple linear regression analysis of intakes of PUFAs in relation to outcomes were performed and adjusted for potential confounders. Results: Absolute n-3 PUFA intake, but not n-3/n-6, was inversely associated with the different measures of body fat. Among n-3 PUFA derivatives, only $\alpha$-linolenic acid (ALA) was inversely associated with body fat measures. No significant interactions with the dietary macronutrient composition were observed. Pro-inflammatory cytokines were not associated with absolute PUFA intake, but the macrophage inflammatory protein-1 $\alpha$ (MIP- $1 \alpha$ ) was associated with the $n-3 / n-6$ ratio. Conclusion: In humans, intake of n-3 PUFAs, in particular ALA, is beneficially associated with body fatness. The favourable association is, however, not reflected in systemic levels of pro-inflammatory cytokines, nor is it influenced by macronutrients in the diet.

(c) 2013 S. Karger GmbH, Freiburg
\end{abstract}


Lund et al.: N-3 Polyunsaturated Fatty Acids, Body Fat and Inflammation

\section{Introduction}

Obesity is the most prevalent nutrition-related disorder in Westernized countries [1]. Obesity, particularly central obesity, is associated with a state of sub-clinical chronic lowgrade systemic inflammation [2,3], induced by abnormal expression and secretion of a variety of circulating pro- and anti-inflammatory biomarkers, the cytokines. These biomarkers are produced by the adipose tissue (AT) and includes tumour necrosis factor alpha (TNF- $\alpha$ ), interleukin-6 (IL-6), interleukin-18 (IL-18) and C-reactive protein (CRP), among others [4-7]. Several studies have shown that the expression and secretion of circulating pro-inflammatory cytokines increase with weight gain and obesity [1,5-12], and decrease markedly with weight loss $[7,8,11,13,14]$. The cytokines secreted from the obese AT are considered to be linked directly to health consequences of obesity, such as insulin resistance (IR) and the metabolic syndrome $[1,2]$. Indeed, increased systemic inflammation is one of the major factors contributing to development of IR [15].

Among nutritional factors with a potential beneficial impact on risk of obesity and related inflammatory processes are n-3 polyunsaturated fatty acids (PUFAs) [16]. The principal n-3 PUFA in Western diet is $\alpha$-linolenic acid (ALA, 18:3(n-3)), which is found in high concentrations in green leafy vegetables, flaxseeds, legumes and nuts, among others [17]. ALA can be further metabolised to the long-chain derivatives, eicosapentaenoic acid (EPA, 20:5(n-3)) and docosahexaenoic acid (DHA, 22:6(n-3)). Dietary EPA and DHA are readily available in significant amounts in cold-water fatty fish such as salmon, mackerel and herring, and even in more concentrated amounts in encapsulated fish oil as supplements [16].

The association between n-3 PUFAs and body composition in healthy adults has been subject to only a few human studies [18], without simultaneous intervention by energyrestricted diet or other life style modification. In a small intervention trial among healthy lean subjects, replacement of $6 \mathrm{~g}$ of visible fat with $6 \mathrm{~g}$ fish oil (n-3 PUFAs) resulted in unchanged body weights, but a significant decrease in body fat mass [19]. Further, in a study among overweight/obese type 2 diabetic women, a moderate dose of n-3 PUFAs was shown to decrease both total fat mass (mainly truncal fat mass) and subcutaneous adipocyte diameter significantly, despite no changes in energy intake [20]. In line with this, studies in rodents have demonstrated that incorporation of n-3 PUFAs, in particular EPA and DHA, into the diet may result in a more favourably obesity phenotype with decreased adipose deposition and reduction in visceral fat, in particular [21-24].

Most common western diets are, however, richer in n- 6 than n-3 PUFAs, and excessive intake of n-6 PUFAs may unfortunately attenuate the beneficial effects of n-3 PUFA. The predominant n-6 PUFA in the diet is linoleic acid (LA, 18:2(n-6)), which is present in vegetable oils, margarines, several fruits and vegetables, nuts, grains and seeds. LA is metabolised to arachidonic acid (AA, 20:4(n-6)) [25].

Further, the macronutrient composition of the diet has been suggested to influence the potential association between PUFAs and anthropometrics. Recently, a study of rodents revealed that the associations between n-6 PUFAs (from corn oil) and body weight as well as AT mass was modified by the balance between carbohydrates and protein in the diet [26]. The n-6 PUFAs were shown to be pro-adipogenic when combined with a diet high in carbohydrates and non-adipogenic when combined with a diet high in protein.

The aim of the present study was to investigate if high intakes of n-3 PUFAs and derivatives hereof among apparently healthy subjects are associated with low total body fat, body fat distribution and systemic pro-inflammatory cytokines, and if such associations were further influenced by the composition of macronutrients in the total diet. 
Lund et al.: N-3 Polyunsaturated Fatty Acids, Body Fat and Inflammation

\section{Material and Methods}

\section{Study Population}

The study population derived from a cohort of 756 complete either monozygotic or dizygotic twin pairs (of both same and opposite sex) born during 1931-1982, who were originally recruited through the population-based nationwide Danish Twin Registry with the purpose of studying the metabolic syndrome and related components $[27,28]$. The age of the twins ranged from 18 to 67 years (mean age 38 years) when they underwent a full 1-day clinical examination within the period of August 1997 to November 2000. At the time of examination, subjects had no known (self-reported) diabetes, cardiovascular disease, pregnancy, breastfeeding, alcohol or drug abuse. The study also included a progressive maximal (oxygen uptake) bicycle test. The Danish Scientific-Ethical Committee and the Danish Data Protection Agency approved the study, and it was conducted according to the principles and guidelines of the Helsinki Declaration in its revised version of 1975 and its amendments of 1983, 1989, and 1996.

\section{Dietary Measurements}

In relation to the clinical examination, participants filled in a comprehensive food frequency questionnaire (FFQ), initially designed for the Danish 'European Prospective Investigation into Cancer and Nutrition' (EPIC) study [29], and validated against two 7-day weighed diet records [30]. The FFQ included 247 foods and recipes, and the subjects were instructed to fill in the frequency of consumption as the number of intakes per day, week or month. The habitual dietary intake reported in the FFQ was based on a 1-month recall and included detailed information regarding: beverage intake, breakfast products, bread and fat on bread, products put on the bread, hot meals, accessories, vegetables, desserts, fruits, cakes, snacks and sweets, type of fat for bread and cooking, cooking style, fast food, meal structure, dietary and organic food habits, and dietary food supplements. Dietary calculations were made using the FoodCalc programme (Jesper Lauritsen, Copenhagen, Denmark) [31] which is based on values from the Danish National Food Tables 1996 [32]. The methods of ascertainment of dietary intake in this study population have been described in detail elsewhere [33]. Dietary information was obtained from 1,212 subjects ( 600 complete twin pairs and 12 incomplete twin pairs).

Based on the data collected from the FFQ, a dietary database was constructed including, among others, total energy intake, macronutrients (given in grams, kilojoules and energy \%), oils, fibre intake and calculations of the dietary intake of n-3 PUFAs and the subgroups ALA, EPA and DHA as well as intake of n-6 PUFAs.

\section{Anthropometric Measurements}

The clinical examination included measurements of height (to the nearest $\mathrm{cm}$ ), weight (measured to the nearest $0.1 \mathrm{~kg}$ on a standing beam scale), waist circumference (WC; measured with a soft tape to the nearest $0.1 \mathrm{~cm}$ at a point in the middle between the lowest rib and the iliac crest) and hip circumference (HC; to the nearest $0.1 \mathrm{~cm}$ measured at the widest part of the glutei region). BMI of each twin was calculated as weight (in kilograms) divided by the square of height (in meters). Further, skin fold thicknesses, to the nearest 0.1 mm, was measured at sub-scapular $(n=1,211)$ and supra-iliac $(n=1,212)$ sites on the right side of the body, using a Harpender calliper. Measurement of skin folds were repeated three times at each measurement point, and the mean was used to reduce risk of measurement error. The truncal skin fold thickness was calculated as the mean of the sub-scapular and supra-iliac skin fold thicknesses $(n=1,211)$. Lastly, body composition was determined by bioelectrical impedance (BI), using a BIA-103 RJL-system analyser (RJL-systems, Detroit, MI, USA) with a $50 \mathrm{kHz}, 800-\mu \mathrm{A}$ device, and the instructions given by the manufacturer was followed. Body fat assessed by BI was estimated using following equation: Fat mass (FM) $=0.819 \times$ weight $-0.064 \times \operatorname{sex} \times$ weight -0.279 (height $\left.^{2} / \mathrm{R} 50\right)-0.231 \times$ height $+0.077 \times$ age +14.941 [34], where R50 is the electrical BI with $50 \mathrm{kHz}$, and where sex is 0 for women and 1 for men $(n=1,019)$. Fat-free mass (FFM) was calculated by subtracting the FM from the body weight. Fat mass index (FMI) and fat free mass index (FFMI) were calculated as FM (kg) / height ${ }^{2}\left(\mathrm{~m}^{2}\right)$ and FFM $(\mathrm{kg}) /$ height $^{2}\left(\mathrm{~m}^{2}\right)$, respectively.

\section{Markers of Inflammation}

In close proximity to the clinical examination, venous blood samples were collected in the morning after an overnight 10- to 12-hour fast. The blood samples were used to measure systemic levels of CRP by ELISA [35] and of the pro-inflammatory cytokines IL-6, IL-18, TNF- $\alpha$, tumour necrosis factor receptor 1 (TNF-R1), and macrophage inflammatory protein 1 alpha (MIP-1 $\alpha$ ) by the Luminex ${ }^{\circledR}$ xMAP technology (Luminex Corporation, Austin, TX, USA) [36]. 
Lund et al.: N-3 Polyunsaturated Fatty Acids, Body Fat and Inflammation

Table 1. Characteristics of the GEMINAKAR study population

\begin{tabular}{|c|c|c|}
\hline & $\begin{array}{l}\text { Number of } \\
\text { observations }\end{array}$ & Mean \pm SD \\
\hline \multicolumn{3}{|l|}{ Demographic variables } \\
\hline Age, years & 1,212 & $37.6 \pm 11.5$ \\
\hline \multicolumn{3}{|l|}{ Anthropometric measurements } \\
\hline Height, m & 1,212 & $173.0 \pm 9.1$ \\
\hline Weight, kg & 1,212 & $73.2 \pm 12.8$ \\
\hline BMI, $\mathrm{kg} / \mathrm{m}^{2}$ & 1,212 & $24.4 \pm 3.5$ \\
\hline $\mathrm{WC}, \mathrm{cm}$ & 1,212 & $84.0 \pm 10.5$ \\
\hline $\mathrm{HC}, \mathrm{cm}$ & 1,212 & $97.6 \pm 8.1$ \\
\hline Skin fold (supra-iliac), mm & 1,212 & $14.7 \pm 7.7$ \\
\hline Skin fold (sub-scapula), mm & 1,211 & $17.5 \pm 7.4$ \\
\hline Total skin fold, $\mathrm{mm}$ & 1,211 & $16.1 \pm 7.0$ \\
\hline Body fat $\%$ & 1,019 & $25.8 \pm 7.8$ \\
\hline FFMI, $\mathrm{kg} / \mathrm{m}^{2}$ & 1,019 & $17.8 \pm 1.8$ \\
\hline FMI, $\mathrm{kg} / \mathrm{m}^{2}$ & 1,019 & $6.5 \pm 2.8$ \\
\hline \multicolumn{3}{|l|}{ Dietary measurements } \\
\hline Energy intake, MJ & 1,212 & $10.0 \pm 3.3$ \\
\hline Carbohydrate, g & 1,212 & $276.5 \pm 98.3$ \\
\hline Protein, g & 1,212 & $94.9 \pm 32.0$ \\
\hline Fat, $g$ & 1,212 & $86.6 \pm 35.7$ \\
\hline$n-3$ PUFAs (sum of all), $g$ & 1,212 & $2.7 \pm 1.4$ \\
\hline n-6 PUFAs (sum of all), $g$ & 1,212 & $10.4 \pm 4.6$ \\
\hline ALA, $g$ & 1,212 & $1.8 \pm 1.1$ \\
\hline EPA, g & 1,212 & $0.2 \pm 0.2$ \\
\hline DHA, g & 1,212 & $0.5 \pm 0.3$ \\
\hline \multicolumn{3}{|l|}{ Pro-inflammatory cytokines } \\
\hline $\mathrm{CRP}, \mu \mathrm{g} / \mathrm{ml}$ & 1,040 & $2.1 \pm 3.7$ \\
\hline $\mathrm{MIP}-1 \alpha, \mu \mathrm{g} / \mathrm{ml}$ & 1,087 & $448.2 \pm 479.7$ \\
\hline IL-6, $\mu \mathrm{g} / \mathrm{ml}$ & 1,088 & $354.6 \pm 585.3$ \\
\hline IL-18, $\mu \mathrm{g} / \mathrm{ml}$ & 1,087 & $492.9 \pm 468.8$ \\
\hline TNF-R1, $\mu \mathrm{g} / \mathrm{ml}$ & 1,088 & $1,794.4 \pm 1,362.4$ \\
\hline $\mathrm{TNF}-\alpha, \mu \mathrm{g} / \mathrm{ml}$ & 1,088 & $48.5 \pm 134.1$ \\
\hline \multicolumn{3}{|l|}{ Other variables } \\
\hline Physical fitness $\left(\mathrm{VO}_{2 \max }\right), \mathrm{l}$ & 1,169 & $2.5 \pm 0.7$ \\
\hline Alcohol intake, $g$ & 1,212 & $13.5 \pm 17.3$ \\
\hline
\end{tabular}

\section{Statistical Analyses}

Descriptive results of the continuous variables are presented in table 1 as mean \pm standard deviation (SD). Preliminary assumption testing was conducted to check for normality, linearity, outliers and homogeneity of variance. The associations between intake of our exposures: n-3 PUFAs, ALA, EPA, DHA, and the n-3/n- 6 ratio and each of the outcomes: BMI, WC, HC, skin folds, body fat \%, FFMI, and FMI, CRP, MIP- $1 \alpha$, IL-6, IL-18, TNF- $\alpha$ and TNF-R1 were investigated using multiple linear regression models adjusted for age and sex (Model 1). The markers of inflammation were not normally distributed, and were log-transformed before further analysis. Secondly, multiple linear regression analyses were performed, adjusting for potential confounders identified in the literature: age, sex, physical fitness as measured by maximal oxygen uptake $\left(\mathrm{VO}_{2 \max }\right)$, current smoking and intake of alcohol (Model 2). Associations were considered statistically significant if $\mathrm{p}<0.05$. When examining interactions between the intake of $n-3$ PUFAs and the macronutrients in the diet, the intake of carbohydrate and protein was divided into high and low intakes, respectively. 
Lund et al.: N-3 Polyunsaturated Fatty Acids, Body Fat and Inflammation

Since the participants in the sample were members of twin pairs and hence to adjust for the mutual dependency of the twin individuals constituting a twin pair, i.e. allowing for twin correlation, a twin cluster method was applied in the analyses.

The statistical analyses were carried out using Stata 9.2 (StataCorp LP, College Station, TX, USA).

\section{Results}

The study population consisted of 1,212 individuals, with a mean age of 37.6 years at the time of the clinical examination (table 1). Subjects had a mean BMI within the normal range $\left(24.4 \pm 3.5 \mathrm{~kg} / \mathrm{m}^{2}\right)$, a mean body fat $\%$ of $25.8 \pm 7.8 \%$ and a mean FMI of $6.5 \pm 2.8 \mathrm{~kg} / \mathrm{m}^{2}$. The mean intake of total $\mathrm{n}-3$ PUFAs was $2.7 \pm 1.4 \mathrm{~g}$ /day while the total intake of $\mathrm{n}-6$ PUFAs was $10.4 \pm 4.6 \mathrm{~g} /$ day. Mean intakes of the investigated sub-groups of the $n-3$ PUFAs appear from table 1. The mean level of CRP was $2.1 \pm 3.7 \mu \mathrm{g} / \mathrm{ml}$.

\section{PUFAs and Body Fat}

Age- and sex-adjusted linear regression analyses revealed inverse associations between intake of n-3 PUFAs and several measures of body fat (Model 1). In Model 1, intake of n-3 PUFAs was significantly associated with BMI, WC, HC and skin folds. Further adjustment for potential confounders (physical fitness, smoking and alcohol intake, i.e., Model 2) resulted in statistically significant inverse associations with all measures of body fat except for FFMI (table 2). The ratio of n-3/n-6 PUFA intake was not significantly associated with measures of body fat (table 2).

\section{ALA, EPA, and DHA and Body Fat}

Similar to what was observed for the overall intake of n-3 PUFAs, ALA was inversely associated with all the anthropometric measures, except for FFMI (Models 1 and 2; table 3). No significant association between intake of EPA and anthropometry was found. Similarly, no significant association between intake of DHA and anthropometry was observed, except for an inverse association between intake of DHA and FFMI in Model 2 (table 3).

\section{PUFAs and Pro-Inflammatory Markers}

No significant associations between consumption of n-3 PUFAs and the assessed markers of inflammation were observed (data not shown). Further, no significant associations with markers of inflammation were found when the separate intake of ALA, EPA and DHA was regarded as exposure. This was also the case when using the ratio of $n-3 / n-6$ as exposure, except for a significant inverse association between intake of $n-3 / n-6$ and MIP- $1 \alpha(\beta-1.37$ $\mu \mathrm{g} / \mathrm{ml} ; 95 \% \mathrm{CI}-2.52 ;-0.22 \mu \mathrm{g} / \mathrm{ml}$ ) (fig. 1). In order to assess whether this was just a by chance observation, we retested the association by use of sub groups of the n-3 PUFAs (ALA, EPA and DHA) divided by $n-6$. A consistent significant inverse association between ALA $/ n-6$ and MIP- $1 \alpha$ was detected ( $\beta-1.93 \mu \mathrm{g} / \mathrm{ml} ; 95 \% \mathrm{CI}-3.68 ;-0.18 \mu \mathrm{g} / \mathrm{ml})$. We did not observe a significant association between MIP- $1 \alpha$ and the selected measures of body fat (data not shown).

Interaction between Macronutrients and Intake of n-3 PUFAs

Regarding the potential impact of intake of macronutrients (protein and carbohydrates) in the diet on the association between n-3 intake and anthropometry, interaction analyses (based on Model 2) revealed no significant associations. 
Lund et al.: N-3 Polyunsaturated Fatty Acids, Body Fat and Inflammation

Table 2. Multiple linear regression models, displaying the associations between the exposures n-3 PUFAs and $n-3 / n-6$, and the anthropometric outcomes

\begin{tabular}{|c|c|c|}
\hline & $\mathrm{n}-3 \beta(95 \% \mathrm{CI})$ & $n-3 / n-6 \beta(95 \% C I)$ \\
\hline \multicolumn{3}{|l|}{ BMI, $\mathrm{kg} / \mathrm{m}^{2}$} \\
\hline Model 1 & $-0.21(-0.35 ;-0.07)^{*}$ & $-0.70(-2.70 ; 1.30)$ \\
\hline Model 2 & $-0.24(-0.38 ;-0.10)^{*}$ & $-0.58(-2.64 ; 1.49)$ \\
\hline \multicolumn{3}{|l|}{$\mathrm{WC}, \mathrm{cm}$} \\
\hline Model 1 & $-0.48(-0.84 ;-0.13)^{*}$ & $-2.57(-7.76 ; 2.62)$ \\
\hline Model 2 & $-0.52(-0.90 ;-0.15)^{*}$ & $-2.50(-7.84 ; 2.84)$ \\
\hline \multicolumn{3}{|l|}{$\mathrm{HC}, \mathrm{cm}$} \\
\hline Model 1 & $-0.35(-0.69 ;-0.02)^{*}$ & $1.17(-3.71 ; 6.05)$ \\
\hline Model 2 & $-0.35(-0.70 ;-0.01)^{*}$ & $2.54(-2.38 ; 7.46)$ \\
\hline \multicolumn{3}{|c|}{ Skin fold (supra-iliac), mm } \\
\hline Model 1 & $-0.45(-0.75 ;-0.15)^{*}$ & $2.03(-2.85 ; 6.90)$ \\
\hline Model 2 & $-0.43(-0.74 ;-0.11)^{*}$ & $1.76(-3.12 ; 6.64)$ \\
\hline \multicolumn{3}{|c|}{ Skin fold (sub-scapula), mm } \\
\hline Model 1 & $-0.53(-0.81 ;-0.25)^{*}$ & $0.22(-3.91 ; 4.35)$ \\
\hline Model 2 & $-0.54(-0.83 ;-0.25)^{*}$ & $-0.47(-4.62 ; 3.69)$ \\
\hline \multicolumn{3}{|c|}{ Total skin fold (truncal), mm } \\
\hline Model 1 & $-0.49(-0.76 ;-0.22)^{*}$ & $1.14(-3.07 ; 5.36)$ \\
\hline Model 2 & $-0.48(-0.77 ;-0.20)^{*}$ & $0.67(-3.46 ; 5.07)$ \\
\hline \multicolumn{3}{|l|}{ Body fat $\%$} \\
\hline Model 1 & $-0.24(-0.51 ; 0.02)$ & $-0.47(-4.10 ; 3.16)$ \\
\hline Model 2 & $-0.31(-0.59 ;-0.03)^{*}$ & $-0.59(-4.33 ; 3.15)$ \\
\hline \multicolumn{3}{|l|}{ FFMI, $\mathrm{kg} / \mathrm{m}^{2}$} \\
\hline Model 1 & $-0.03(-0.09 ; 0.02)$ & $-0.49(-1.25 ; 0.26)$ \\
\hline Model 2 & $-0.04(-0.10 ; 0.02)$ & $-0.41(-1.21 ; 0.40)$ \\
\hline \multicolumn{3}{|l|}{ FMI, $\mathrm{kg} / \mathrm{m}^{2}$} \\
\hline Model 1 & $-0.09(-0.20 ; 0.01)$ & $-0.14(-1.70 ; 1.42)$ \\
\hline Model 2 & $-0.11(-0.22 ;-0.01)^{*}$ & $-0.13(-1.73 ; 1.47)$ \\
\hline
\end{tabular}

Model 1: adjusted for age and sex.

Model 2: adjusted for age, sex, physical fitness, smoking and alcohol intake.

${ }^{*}$ Confidence interval excluding $0(\mathrm{p}<0.05)$.

Fig. 1. Inverse association between intake of n-3/n- 6 ratio and MIP- $1 \alpha$.

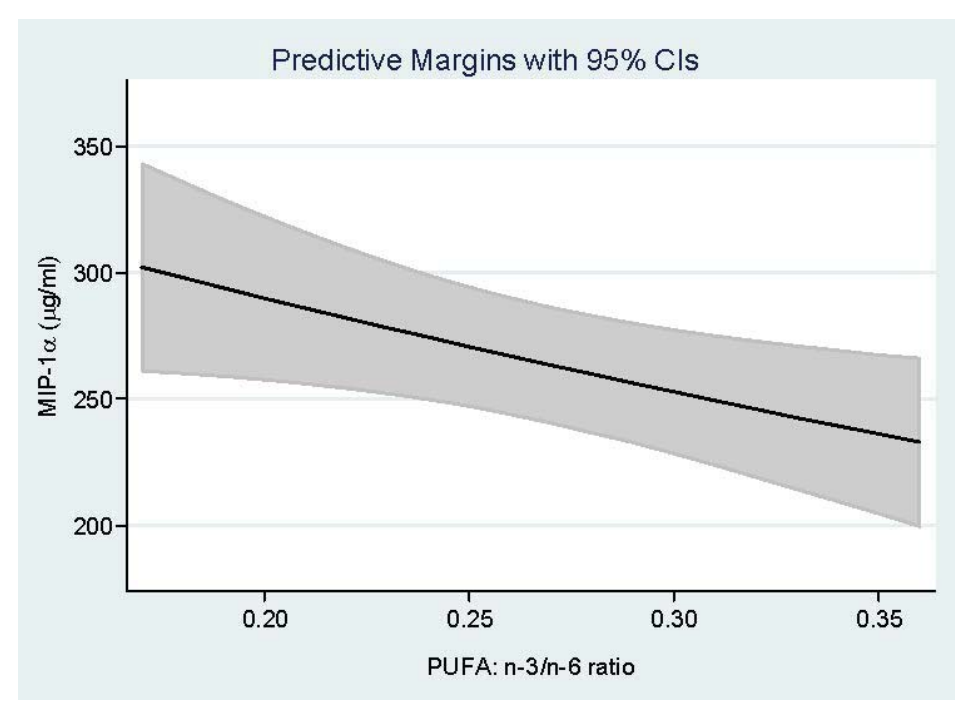


Lund et al.: N-3 Polyunsaturated Fatty Acids, Body Fat and Inflammation

Table 3. Multiple linear regression models, displaying the associations between the exposures ALA, EPA, DHA and the anthropometric outcomes

\begin{tabular}{|c|c|c|c|}
\hline & ALA $\beta(95 \%$ CI $)$ & EPA $\beta(95 \%$ CI $)$ & DHA $\beta(95 \%$ CI $)$ \\
\hline \multicolumn{4}{|l|}{ BMI, $\mathrm{kg} / \mathrm{m}^{2}$} \\
\hline Model 1 & $-0.31(-0.49 ;-0.12)^{*}$ & $-0.27(-1.28 ; 0.73)$ & $-0.37(-0.95 ; 0.21)$ \\
\hline Model 2 & $-0.31(-0.45 ;-0.13)^{*}$ & $-0.60(-1.71 ; 0.51)$ & $-0.58(-1.18 ; 0.03)$ \\
\hline \multicolumn{4}{|l|}{$\mathrm{WC}, \mathrm{cm}$} \\
\hline Model 1 & $-0.60(-1.06 ;-0.14)^{*}$ & $-1.51(-4.22 ; 1.20)$ & $-1.50(-3.03 ; 0.03)$ \\
\hline Model 2 & $-0.62(-1.10 ;-0.15)^{*}$ & $-1.67(-4.91 ; 1.58)$ & $-1.73(-3.46 ; 0.01)$ \\
\hline \multicolumn{4}{|c|}{ (2) } \\
\hline Model 1 & $-0.65(-1.08 ;-0.22)^{*}$ & $1.05(-1.36 ; 3.45)$ & $0.26(-1.13 ; 1.65)$ \\
\hline Model 2 & $-0.64(-1.07 ;-0.21)^{*}$ & $1.53(-0.93 ; 4.00)$ & $0.32(-1.12 ; 1.77)$ \\
\hline \multicolumn{4}{|c|}{ Skin fold (supra-iliac), mm } \\
\hline Model 1 & $-0.78(-1.16 ;-0.41)^{*}$ & $0.76(-1.97 ; 3.50)$ & $0.00(-1.42 ; 1.42)$ \\
\hline Model 2 & $-0.81(-1.20 ;-0.42)^{*}$ & $2.01(-0.87 ; 4.88)$ & $0.57(-0.90 ; 2.03)$ \\
\hline \multicolumn{4}{|c|}{ Skin fold (sub-scapula), mm } \\
\hline Model 1 & $-0.72(-1.09 ;-0.35)^{*}$ & $-1.28(-3.47 ; 0.91)$ & $-1.06(-2.27 ; 0.14)$ \\
\hline Model 2 & $-0.77(-1.15 ; 0.39)^{*}$ & $-0.59(-2.84 ; 1.65)$ & $-0.76(-1.99 ; 0.47)$ \\
\hline \multicolumn{4}{|c|}{ Total skin fold (truncal), mm } \\
\hline Model 1 & $-0.75(-1.10 ;-0.41)^{*}$ & $-0.26(-2.58 ; 2.06)$ & $-0.54(-1.76 ; 0.69)$ \\
\hline Model 2 & $-0.79(-1.15 ;-0.23)^{*}$ & $0.70(-1.67 ; 3.08)$ & $-0.10(-1.35 ; 1.14)$ \\
\hline \multicolumn{4}{|l|}{ Body fat $\%$} \\
\hline Model 1 & $-0.42(-0.78 ;-0.07)^{*}$ & $0.46(-1.25 ; 2.17)$ & $-0.07(-1.12 ; 0.98)$ \\
\hline Model 2 & $-0.47(-0.84 ;-0.11)^{*}$ & $0.11(-1.90 ; 2.11)$ & $-0.37(-1.51 ; 0.78)$ \\
\hline \multicolumn{4}{|l|}{ FFMI, $\mathrm{kg} / \mathrm{m}^{2}$} \\
\hline Model 1 & $-0.03(-0.11 ; 0.04)$ & $-0.17(-0.56 ; 0.23)$ & $-0.14(-0.37 ; 0.08)$ \\
\hline Model 2 & $-0.03(-0.10 ; 0.05)$ & $-0.33(-0.74 ; 0.09)$ & $-0.24(-0.47 ;-0.01)^{*}$ \\
\hline \multicolumn{4}{|l|}{ FMI, $\mathrm{kg} / \mathrm{m}^{2}$} \\
\hline Model 1 & $-0.15(-0.28 ;-0.01)^{*}$ & $0.06(-0.68 ; 0.79)$ & $-0.12(-0.55 ; 0.31)$ \\
\hline Model 2 & $-0.16(-0.30 ;-0.02)^{*}$ & $-0.09(-0.93 ; 0.76)$ & $-0.23(-0.70 ; 0.22)$ \\
\hline
\end{tabular}

Model 1: adjusted for age and sex.

Model 2: adjusted for age, sex, physical fitness, smoking and alcohol intake.

*Confidence interval excluding $0(\mathrm{p}<0.05)$.

\section{Discussion}

In the present study of 1,212 Danish adults with detailed information on food intake, anthropometric measures and levels of pro-inflammatory markers, we found that high intakes of n-3 PUFAs and in particular ALA were associated with lower levels of body fat measured as BMI, WC, HC, truncal skin folds, body fat \%, and FMI, whereas no association was found for FFMI. Macronutrient composition of the diet did not influence these associations. The association between intake of PUFAs and anthropometry was not paralleled by a beneficial effect on pro-inflammatory markers.

A recent comprehensive review of the impact of diet and physical activity on obesity in humans [37] did not evaluate the effect of n-3 PUFAs on fatness, apparently due to lack of observational studies on the subject. However, several studies in rodents have shown that n-3 PUFAs may influence body composition and obesity. These studies have shown that increased intake of n-3 PUFAs may decrease fat accumulation, mainly visceral fat, when fed on a high-fat $\operatorname{diet}[22,24]$ and reduce body weight when already obese [38]. The mechanisms by which n-3 PUFAs are proposed to influence body fat in a positive manner are not well understood [18]. It has been suggested that n-3 PUFAs, in particular EPA and DHA, may activate a metabolic 
Lund et al.: N-3 Polyunsaturated Fatty Acids, Body Fat and Inflammation

change in adipocytes including an increased $\beta$-oxidation and suppressed lipogenesis in abdominal fat [21], that n-3 PUFAs may reduce fat accumulation via a reduced adipocyte hypertrophy [22, 24, 39] or that n-3 PUFAs may induce apoptosis in the AT [22]. In addition, the balance between energy intake and expenditure may be affected in a positive manner by intake of n-3 PUFAs. In a study with high-fat fed rats, supplementation with EPA resulted in a decrease in food intake [22]. Still, further studies in humans are warranted to clarify the mechanisms and to confirm the potential health-promoting effects of n-3 PUFAs on obesity.

In the present study, we did not find any consistent significant associations between intake of either EPA or DHA and anthropometric outcomes. These findings are in contrast to other findings from the literature. For instance, in a cross-sectional study of normal-weight, overweight and obese men and women, a significant inverse association was found between plasma concentrations of n-3 PUFAs and BMI, WC as well as HC. In addition, another study found that total n-3 PUFAs, EPA, and DHA were inversely associated with BMI, WC and HC in the obese subjects [40]. The lack of associations in the present study may be due to the low mean intakes of EPA and DHA as well as the low variation in intakes in the study population.

In contrast to the above mentioned studies, including the present, where beneficial associations between n-3 PUFAs and anthropometry were observed, a study of 26 overweight or moderately obese men and women did not show any significant association between a diet rich in n-3 PUFAs and body weight, fat mass as well as energy expenditure [41, 42].

The balance between intake of total n-3 and n-6 PUFAs has been suggested to be a more important determinant of health, especially cardiovascular health, than the absolute intake of $n-3$ and n-6 PUFAs. A low ratio of $n-3 / n-6$, resulting either from a very low intake of $n-3$ PUFAs and/or excessive intake of n-6 PUFAs is a potential risk factor for cardiovascular disease and inflammatory disorders [43]. The optimal ratio of n-3 to n-6 PUFAs in a balanced healthy diet has been proposed to be $1: 4$, or even closer to 1 , but in western diets the ratio varies in the range between 1:15 and 1:20 [43]. We also investigated if the ratio of $n-3 / n-6$ PUFAs was associated with obesity phenotypes, but did not observe any associations. The ratio of n-3/n-6 PUFAs in the present study was almost 1:4, what is quite unusual for a typical western diet, and this could be a possible reason for why we did not observe any associations. Alternatively, as for many other non-energy-yielding nutrients, the absolute intake of n-3 PUFAs and ALA may be more important to consider in a healthy diet than the ratio with $n-6$ in relation to development of obesity. Further and preferably longitudinal studies in humans - also of the relation between n- 6 intakes per se and fatness among humans - should be undertaken to elucidate this aspect.

Regarding the potential impact of macronutrient composition in the diet on the influence of PUFAs on obesity [26], we examined the hypothesis that a diet high in carbohydrates may negatively influence the advantageous properties of n-3 PUFAs in relation to obesity, whereas a diet high in protein may enhance the beneficial influence of an increased intake of n-3 PUFAs, resulting in a lower grade of obesity. However, our analyses revealed no interactions between macronutrients and n-3 PUFAs in relation to anthropometry. Hence, further studies in humans are warranted to confirm or reject the hypothesis generated from animal experiments.

In the present study, a tendency towards a positive effect of intake of n-3 PUFAs on MIP-1 $\alpha$ was observed. The lack of an effect on typical markers of inflammation, such as CRP and TNF- $\alpha$, probably could be explained by the fact that, with a mean BMI within the normal range, too few individuals had measurable obesity-related systemic low-grade inflammation. In a study of overweight subjects (BMI $28.6 \pm 10.4 \mathrm{~kg} / \mathrm{m}^{2}$ ), the secretion of pro-inflammatory cytokines from abdominal subcutaneous AT was significantly elevated in very large adipocytes compared with small or medium adipocytes from the same individual [44]. MIP-1 $\alpha$ is one of a number of chemokines more recently shown to correlate positively to central fat mass [45], but to our knowledge no previous studies have assessed the potential effect of nutrients on levels of 
MIP- $1 \alpha$. While we did observe a persisting association between MIP- $1 \alpha$ and not only $n-3 / n-6$ ratio but also subgroups hereof, we did not confirm a strong association between MIP-1 $\alpha$ and measures of fat mass. Both of these new observations warrant further investigation.

The primary strength of the present study was the assessment of an unselected study population counting more than 1,000 individuals and with more anthropometric measurements than in any of the few previous studies of the impact of PUFAs on obesity in humans. Further, we had detailed information on habitual dietary intake as well as on a number of potential confounders.

A potential limitation to the study was the cross-sectional design, which did not allow us to test the temporal direction between intake of PUFAs and body fat mass and body fat distribution. However, in line with evidence from animal studies, it seems more likely that low $n-3$ PUFA intakes result in fatness, rather than the opposite scenario, where fatness should lead to a decrease in intake of n-3 PUFA. Another potential limitation of the study was the estimation of dietary intakes of n-3 and n-6 PUFAs based on self-administrated FFQ, which in a validation using dietary records for comparison has shown to underestimate intakes of total energy, protein and PUFAs. However, this observation would only bias associations in the present study if reporting of intake of PUFAs depended on the level of obesity and that is more likely the case for intake of for example saturated fatty acids. Also, underreporting, if present, would only be expected to limit the interpretation of absolute levels of PUFA intake. Lastly, we acknowledge that analyses are based on a study population assessed almost a decade ago. However, despite potential changes in content of PUFAs in foods since then, physiological relations between PUFAs and fatness (factors which were assessed simultaneously) are not likely to have changed over time.

In conclusion, the present study in humans suggests that intake of both total n-3 PUFAs and ALA is inversely associated with most measures of body fat and level of MIP-1 $\alpha$, but notably not with other markers of systematic inflammation among healthy subjects. We could not confirm the hypothesis that increased intake of protein enhances the beneficial effects of n-3 PUFAs on anthropometry or that increased intake of carbohydrate eliminates these effects. Follow-up studies in humans are needed to confirm the beneficial effect of n-3 PUFAs on fatness and to further address a potential effect-modifying role of macronutrients in the diet.

\section{Acknowledgement}

This study was carried out as a part of the research program of the Danish Obesity Research Centre (DanORC, see www.danorc.dk). DanORC is supported by the Danish Council for Strategic Research (Grant 2101-06-0005). Dr. Tine Jess was supported by a Female research Leader grant from the Danish Council of Independent Research (Grant 09-066323). The GEMINAKAR project was supported by grants from the Medical Research Fund, the Danish Diabetes Association, the NOVO Foundation, the Danish Heart Foundation, and Apotekerfonden. Dr. Kirsten Ohm Kyvik has obtained supports from the European Union Contract No. QLG2-CT-2002-01254. The present study was supported by the DIOGENES which is the acronym for 'Diet, Obesity and Genes' supported by the European Community (Contract no. FP6-513946), see www.diogenes-eu. org. We sincerely thank our statistician Lars Ängquist, PhD, for his contribution to the additional analyses.

\section{Authors' Contribution}

A.S.Q.L and T.J. initiated the study, which was based on the GEMINAKAR cohort originally initiated by K.K. and T.I.A.S. K.S. and D.M.H. carried out analyses of inflammatory markers. M.G. performed the statistical analyses. A.S.Q.L., T.J. and M.G. interpreted the results and A.S.Q.L. drafted the first version of the manuscript. A.L.H. performed further statistical analyses and updated the manuscript. The manuscript was critically revised by all co-authors. All authors accepted the final version of the manuscript. 
Lund et al.: N-3 Polyunsaturated Fatty Acids, Body Fat and Inflammation

\section{Disclosure Statement}

The authors declare no conflicts of interest in relation to the present study.

\section{References}

1 Trayhurn P: Endocrine and signalling role of adipose tissue: new perspectives on fat. Acta Physiol Scand 2005; 184:285-293.

2 Hotamisligil GS: Inflammation and metabolic disorders. Nature 2006;444:860-867.

- 3 Horng T, Hotamisligil GS. Linking the inflammasome to obesity-related disease. Nat Med 2011;17:164-165.

4 Rajala MW, Scherer PE: Minireview: the adipocyte - at the crossroads of energy homeostasis, inflammation, and atherosclerosis. Endocrinology 2003;144:3765-3773.

-5 Trayhurn P, Wood IS: Adipokines: inflammation and the pleiotropic role of white adipose tissue. Br J Nutr 2004; $92: 347-355$

6 Kern PA, Ranganathan S, Li C, Wood L, Ranganathan G: Adipose tissue tumor necrosis factor and interleukin-6 expression in human obesity and insulin resistance. Am J Physiol Endocrinol Metab 2001; 280:E745-E751.

7 Hotamisligil GS, Arner P, Caro JF, Atkinson RL, Spiegelman BM: Increased adipose tissue expression of tumor necrosis factor-alpha in human obesity and insulin resistance. J Clin Invest 1995;95:2409-2415.

-8 Kern PA, Saghizadeh M, Ong JM, Bosch RJ, Deem R, Simsolo RB: The expression of tumor necrosis factor in human adipose tissue. Regulation by obesity, weight loss, and relationship to lipoprotein lipase. J Clin Invest 1995;95:2111-2119.

-9 Maachi M, Pieroni L, Bruckert E, Jardel C, Fellahi S, Hainque B, Capeau J, Bastard JP. Systemic low-grade inflammation is related to both circulating and adipose tissue TNFalpha, leptin and IL-6 levels in obese women. Int J Obes Relat Metab Disord 2004;28:993-997.

10 Fain JN, Madan AK, Hiler ML, Cheema P, Bahouth SW: Comparison of the release of adipokines by adipose tissue, adipose tissue matrix, and adipocytes from visceral and subcutaneous abdominal adipose tissues of obese humans. Endocrinology 2004;145:2273-2282.

-11 Ziccardi P, Nappo F, Giugliano G, Esposito K, Marfella R, Cioffi M, D’Andrea F, Molinari AM, Giugliano D: Reduction of inflammatory cytokine concentrations and improvement of endothelial functions in obese women after weight loss over one year. Circulation 2002;105:804-809.

-12 Panagiotakos DB, Pitsavos C, Yannakoulia M, Chrysohoou C, Stefanadis C: The implication of obesity and central fat on markers of chronic inflammation: the ATTICA study. Atherosclerosis 2005;183:308-315.

13 Ryan AS, Nicklas BJ: Reductions in plasma cytokine levels with weight loss improve insulin sensitivity in overweight and obese postmenopausal women. Diabetes Care 2004;27:1699-1705.

14 Bastard JP, Jardel C, Bruckert E, Blondy P, Capeau J, Laville M, Vidal H, Hainque B: Elevated levels of interleukin 6 are reduced in serum and subcutaneous adipose tissue of obese women after weight loss. J Clin Endocrinol Metab 2000;85:3338-3342.

-15 Fedor D, Kelley DS: Prevention of insulin resistance by n-3 polyunsaturated fatty acids. Curr Opin Clin Nutr Metab Care 2009;12:138-146.

16 Robinson LE, Buchholz AC, Mazurak VC: Inflammation, obesity, and fatty acid metabolism: influence of n-3 polyunsaturated fatty acids on factors contributing to metabolic syndrome. Appl Physiol Nutr Metab 2007;32: 1008-1024.

17 Burdge GC, Calder PC: Conversion of alpha-linolenic acid to longer-chain polyunsaturated fatty acids in human adults. Reprod Nutr Dev 2005;45:581-597.

18 Buckley JD, Howe PR: Anti-obesity effects of long-chain omega-3 polyunsaturated fatty acids. Obes Rev 2009; 10:648-659.

19 Couet C, Delarue J, Ritz P, Antoine JM, Lamisse F: Effect of dietary fish oil on body fat mass and basal fat oxidation in healthy adults. Int J Obes Relat Metab Disord 1997;21:637-643.

-20 Kabir M, Skurnik G, Naour N, Pechtner V, Meugnier E, Rome S, Quignard-Boulange A, Vidal H, Slama G, Clement K, Guerre-Millo M, Rizkalla SW: Treatment for 2 mo with $n 3$ polyunsaturated fatty acids reduces adiposity and some atherogenic factors but does not improve insulin sensitivity in women with type 2 diabetes: a randomized controlled study. Am J Clin Nutr 2007;86:1670-1679.

-21 Flachs P, Horakova 0, Brauner P, Rossmeisl M, Pecina P, Franssen-van Hal N, Ruzickova J, Sponarova J, Drahota Z, Vlcek C, Keijer J, Houstek J, Kopecky J: Polyunsaturated fatty acids of marine origin upregulate mitochondrial biogenesis and induce beta-oxidation in white fat. Diabetologia 2005;48:2365-2375.

-22 Perez-Matute P, Perez-Echarri N, Martinez JA, Marti A, Moreno-Aliaga MJ: Eicosapentaenoic acid actions on adiposity and insulin resistance in control and high-fat-fed rats: role of apoptosis, adiponectin and tumour necrosis factor-alpha. Br J Nutr 2007;97:389-398.

-23 Peyron-Caso E, Quignard-Boulange A, Laromiguiere M, Feing-Kwong-Chan S, Veronese A, Ardouin B, Slama G, Rizkalla SW: Dietary fish oil increases lipid mobilization but does not decrease lipid storage-related enzyme activities in adipose tissue of insulin-resistant, sucrose-fed rats. J Nutr 2003;133:2239-2243. 
Lund et al.: N-3 Polyunsaturated Fatty Acids, Body Fat and Inflammation

24 Ruzickova J, Rossmeisl M, Prazak T, Flachs P, Sporanova J, Veck M, Tvrzicka E, Bryhn M, Kopecky J: Omega-3 PUFA of marine origin limit diet-induced obesity in mice by reducing cellularity of adipose tissue. Lipids 2004; 39:1177-1185.

25 Calder PC: Polyunsaturated fatty acids, inflammatory processes and inflammatory bowel diseases. Mol Nutr Food Res 2008;52:885-897.

-26 Madsen L, Pedersen LM, Liaset B, Ma T, Petersen RK, van den Berg S, Pan J, Müller-Decker K, Dülsner ED, Kleemann R, Kooistra T, Døskeland SO, Kristiansen K: cAMP-dependent signaling regulates the adipogenic effect of n-6 polyunsaturated fatty acids. J Biol Chem 2008;283:7196-7205.

-27 Schousboe K, Visscher PM, Henriksen JE, Hopper JL, Sørensen TIA, Kyvik KO: Twin study of genetic and environmental influences on glucose tolerance and indices of insulin sensitivity and secretion. Diabetologia 2003; 46:1276-1283.

-28 Schousboe K, Visscher PM, Erbas B, Kyvik KO, Hopper JL, Henriksen JE, Heitmann BL, Sørensen TIA: Twin study of genetic and environmental influences on adult body size, shape, and composition. Int J Obes Relat Metab Disord 2004;28:39-48.

29 Overvad K, Tjonneland A, Haraldsdottir J, Ewertz M, Jensen OM: Development of a semiquantitative food frequency questionnaire to assess food, energy and nutrient intake in Denmark. Int J Epidemiol 1991;20: 900-905.

-30 Tjonneland A, Overvad K, Haraldsdottir J, Bang S, Ewertz M, Jensen OM: Validation of a semiquantitative food frequency questionnaire developed in Denmark. Int J Epidemiol 1991;20:906-912.

31 Lauritsen J: FoodCalc version 1.3. www.ibt dk/jesper/foodcalc. 2007 (last accessed July 30, 2013).

32 Møller A, Saxholt E. Levnedmiddeltabeller (Food composition tables). 4 edn. Copenhagen, Levnedsmiddelstyrelsen (National Food Agency), 1996.

-33 Hasselbalch AL, Heitmann BL, Kyvik KO, Sørensen TIA: Studies of twins indicate that genetics influence dietary intake. J Nutr 2008;138:2406-2412.

34 Heitmann BL: Evaluation of body fat estimated from body mass index, skinfolds and impedance. A comparative study. Eur J Clin Nutr 1990;44:831-837.

-35 Skogstrand K, Ekelund CK, Thorsen P, Vogel I, Jacobsson B, Nørgaard-Pedersen B, Hougaard DM: Effects of blood sample handling procedures on measurable inflammatory markers in plasma, serum and dried blood spot samples. J Immunol Methods 2008;336:78-84.

-36 Skogstrand K, Thorsen P, Norgaard-Pedersen B, Schendel DE, Sorensen LC, Hougaard DM: Simultaneous measurement of 25 inflammatory markers and neurotrophins in neonatal dried blood spots by immunoassay with xMAP technology. Clin Chem 2005;51:1854-1866.

37 Summerbell CD, Douthwaite W, Whittaker V, Ells LJ, Hiller F, Smith S, Kelly S, Edmunds LD, Macdonald I: The association between diet and physical activity and subsequent excess weight gain and obesity assessed at 5 years of age or older: a systematic review of the epidemiological evidence. Int J Obes (Lond) 2009;33(suppl 3):S1-92.

38 Huang XF, Xin X, McLennan P, Storlien L: Role of fat amount and type in ameliorating diet-induced obesity: insights at the level of hypothalamic arcuate nucleus leptin receptor, neuropeptide $Y$ and pro-opiomelanocortin mRNA expression. Diabetes Obes Metab 2004;6:35-44.

-39 Raclot T, Groscolas R, Langin D, Ferre P: Site-specific regulation of gene expression by n-3 polyunsaturated fatty acids in rat white adipose tissues. J Lipid Res 1997;38:1963-1972.

40 Micallef MA, Munro IA, Garg ML: An inverse relationship between plasma n-3 fatty acids and C-reactive protein in healthy individuals. Eur J Clin Nutr 2009;63:1154-1156.

-41 Kratz M, Callahan HS, Yang PY, Matthys CC, Weigle DS: Dietary n-3-polyunsaturated fatty acids and energy balance in overweight or moderately obese men and women: a randomized controlled trial. Nutr Metab (Lond) 2009;6:24.

42 Kratz M, Swarbrick MM, Callahan HS, Matthys CC, Havel PJ, Weigle DS: Effect of dietary n-3 polyunsaturated fatty acids on plasma total and high-molecular-weight adiponectin concentrations in overweight to moderately obese men and women. Am J Clin Nutr 2008;87:347-353.

$\checkmark 43$ Simopoulos AP: Evolutionary aspects of diet, the omega-6/omega-3 ratio and genetic variation: nutritional implications for chronic diseases. Biomed Pharmacother 2006;60:502-507.

44 Skurk T, Berti-Huber C, Herder C, Hauner H: Relationship between adipocyte size and adipokine expression and secretion. J Clin Endocrinol Metab 2007;92:1023-1033.

45 Glintborg D, Andersen M, Richelsen B, Bruun JM: Plasma monocyte chemoattractant protein-1 (MCP-1) and macrophage inflammatory protein-1alpha are increased in patients with polycystic ovary syndrome (PCOS) and associated with adiposity, but unaffected by pioglitazone treatment. Clin Endocrinol (Oxf) 2009;71:652658. 